\title{
Strain-induced partially flat band, helical snake states, and interface superconductivity in topological crystalline insulators
}

\author{
Evelyn Tang ${ }^{1}$ and Liang $\mathrm{Fu}^{1}$ \\ ${ }^{1}$ Department of Physics, Massachusetts Institute of Technology, Cambridge, MA 02139
}

\begin{abstract}
Topological crystalline insulators in IV-VI compounds host novel topological surface states consisting of multi-valley massless Dirac fermions at low energy. Here we show that strain generically acts as an effective gauge field on these Dirac fermions and creates pseudo-Landau orbitals without breaking time-reversal symmetry. We predict the realization of this phenomenon in IV-VI semiconductor heterostructures, due to a naturally occurring misfit dislocation array at the interface that produces a periodically varying strain field. Remarkably, the zero-energy Landau orbitals form a flat band in the vicinity of the Dirac point, and coexist with a network of snake states at higher energy. We propose that the high density of states of this flat band gives rise to interface superconductivity observed in IV-VI semiconductor multilayers at unusually high temperatures, with non-BCS behavior. Our work demonstrates a new route to altering macroscopic electronic properties to achieve a partially flat band, and paves the way for realizing novel correlated states of matter.
\end{abstract}

The recently discovered topological crystalline insulators host novel topological surface states that are protected by the symmetry of the underlying crystal [1 4 . At low carrier energy, these surface states consist of multi-valley massless Dirac fermions, whose characteristic properties are highly tunable by external perturbations. Breaking the crystal symmetry at the atomic scale generates a Dirac mass and leads to gapped phases [1, [5] with potentially novel functionalities in low-power electronics and spintronics 6 [8.

The (001) surface states of topological crystalline insulators $\mathrm{SnTe}$ and $\mathrm{Pb}_{1-x} \mathrm{Sn}_{x} \mathrm{Te}(\mathrm{Se})$ consist of massless Dirac fermions at four valleys that exhibit spin texture of the same chirality [1]. The four Dirac points are located at two pairs of opposite momenta, denoted by $\pm \mathbf{K}_{1}$ and $\pm \mathbf{K}_{2}$, in the vicinity of the $X_{1}$ and $X_{2}$ point in the surface Brillouin zone respectively. $\mathbf{K}_{1}$ and $\mathbf{K}_{2}$ are related by four-fold rotation around the surface normal, while $+\mathbf{K}_{j}$ and $-\mathbf{K}_{j}$ are related by time-reversal symmetry (see Fig.1a). Importantly, unlike in the case of topological insulators 9 11, the Dirac points in topological crystalline insulators are not pinned at timereversal-invariant momenta 12 , because their massless nature is protected by crystal symmetry instead of time reversal[13, 14. As a consequence, perturbations can move such Dirac points in momentum space, mimicking the effect of a gauge field vector potential without breaking time reversal symmetry. Two effective ways of moving surface Dirac points in topological crystalline insulators are (i) alloy composition tuning, as recently demonstrated in $\mathrm{Pb}_{1-x} \mathrm{Sn}_{x} \mathrm{Te}(\mathrm{Se})[15$ and (ii) strain, which is the subject of this work.

We first use symmetry analysis to determine the general form of strain-induced Dirac point displacement on the topological crystalline insulator (001) surface, which is equivalent to a strain-induced gauge field vector potential $\mathbf{A}_{j} \equiv \mathbf{K}_{j}^{\prime}-\mathbf{K}_{j}$ acting on the Dirac fermion at valley $\mathbf{K}_{j}$. To lowest order, $\mathbf{A}_{j}=\left(A_{j}^{x}, A_{j}^{y}\right)$ is linearly proportional to the strain field $u_{i j}$ as given by (see Methods):

$$
\begin{aligned}
& \mathbf{A}_{1}=\left(\alpha_{1} u_{x x}+\alpha_{2} u_{y y}, \alpha_{3} u_{x y}\right), \\
& \mathbf{A}_{2}=\left(\alpha_{3} u_{x y}, \alpha_{1} u_{y y}+\alpha_{2} u_{x x}\right) .
\end{aligned}
$$

where $u_{i j} \equiv\left(\partial_{j} u_{i}+\partial_{i} u_{j}\right) / 2$ is the spatial gradient of the displacement field $\mathbf{u}$, and the coordinate axes $x$ and $y$ are along the [110] and [110] directions respectively. $\alpha_{1}, \alpha_{2}$ and $\alpha_{3}$ denote three independent coupling constants. As shown in Fig.1, under a given type of strain, the Dirac fermions at valleys $\mathbf{K}_{1}$ and $\mathbf{K}_{2}$ experience distinct gauge fields $\mathbf{A}_{1}$ and $\mathbf{A}_{2}$. In addition, opposite gauge fields $-\mathbf{A}_{j}$ are induced at the Dirac valleys $-\mathbf{K}_{j}$ as required by time reversal symmetry.

A uniform strain can only produce a constant gauge field vector potential, which yields zero pseudo-magnetic field $\mathbf{B} \equiv \nabla \times \mathbf{A}$. Instead, a highly inhomogeneous strain is required to create a strong pseudo-magnetic field, which is difficult to engineer with high precision and control. Such a field was previously observed in graphene nanobubbles 16 19, which are however localized within nanoscale regions thus leaving electronic properties on a large scale essentially unaltered.

Here we show that a periodic pseudo-magnetic field covering macroscopic spatial regions arises naturally in IV-VI semiconductor (001) heterostructures consisting of a topological crystalline insulator and a trivial insulator. This field is created by misfit dislocations that are known to spontaneously form at the interface due to lattice mismatch. Remarkably, these dislocations self-organize into a nearly perfect two-dimensional square array with a period of 3-25nm (see Fig.2), as observed by transmission electron spectroscopy, X-ray diffraction, and scanning tunneling microscopy in $\mathrm{SnTe} / \mathrm{PbTe}, \mathrm{PbTe} / \mathrm{PbS}$, $\mathrm{PbTe} / \mathrm{PbSe}$, and $\mathrm{PbTe} / \mathrm{YbS} 20$ 22]. This dislocation superstructure naturally produces an inhomogeneous strain 

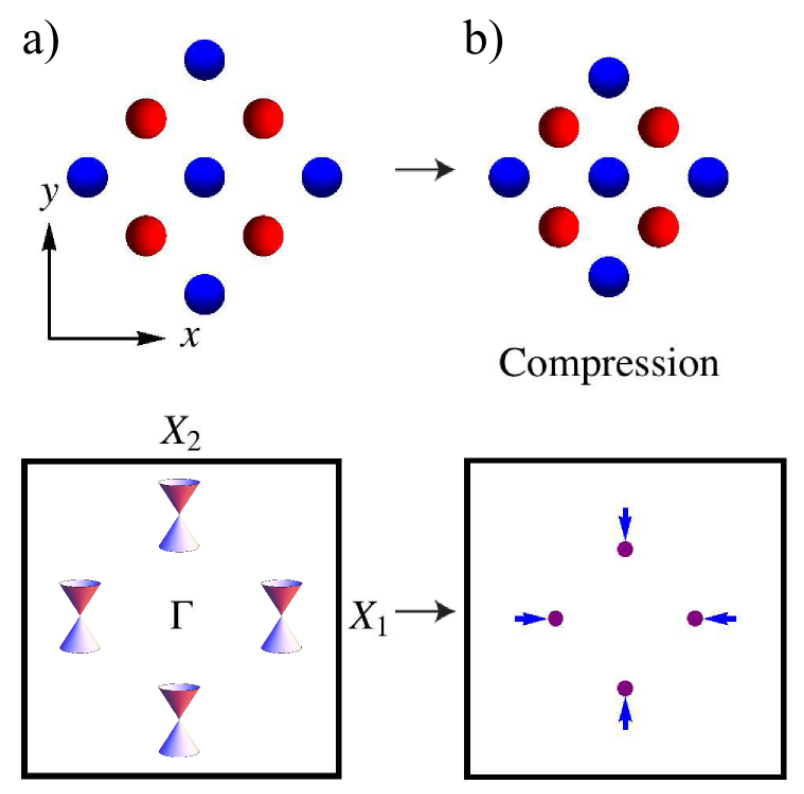

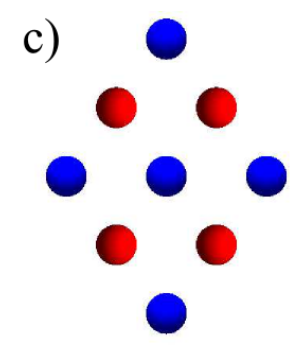

Uniaxial stretch

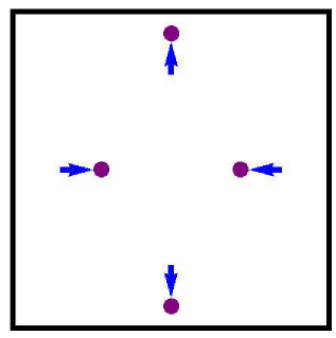

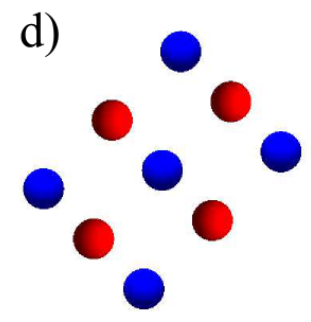

Shear

FIG. 1. Strain-induced Dirac point shift on the (001) surface of a topological crystalline insulator such as SnTe and $\mathbf{P b}_{1-x} \mathbf{S n}_{x} \mathbf{S e}(\mathbf{T e})$. Top: strain on the rocksalt structure of a) compression, b) uniaxial stretch and c) shear; the $x$ and $y$ axes are in the (110) and (110) directions respectively. Bottom: the corresponding strain-induced shift (arrows) of Dirac points to new positions (circles) in the (001) surface Brillouin zone, which is equivalent to an effective gauge field.

field, which gives rise to an unusual pseudo-magnetic field $\mathbf{B}(x, y)$ that alternates with a nanoscale period and averages to zero. Instead, it should be pointed out that the widely studied uniform pseudo-magnetic field is physically impossible in the thermodynamic limit, due to the bounded nature of strain.

To calculate $\mathbf{B}(x, y)$, we first note the experimental observation 22 that the dislocation array is a superposition of two sets of equally spaced parallel dislocation lines along the $x$ and $y$ directions respectively. Each line is an edge dislocation with a Burgers vector $\mathbf{b}=\frac{a}{2} \hat{z} \times \hat{e}$ ( $a$ is the lattice constant), which is parallel to the interface and perpendicular to the line direction $\hat{e}=\hat{x}$ or $\hat{y}$. The set of dislocation lines along the $y$ direction creates a displacement field at the interface. The corresponding strain field contains two in-plane components $u_{x x}$ and $u_{y y}$ that periodically vary in the $x$ direction. $u_{x x}$ and $u_{y y}$ are the sum of strains from each dislocation line indexed by $N$ :

$$
\begin{aligned}
& u_{x x}(x)=\sum_{N} u_{x x}^{0}(x-N \lambda), \\
& u_{y y}(x)=\sum_{N} u_{y y}^{0}(x-N \lambda),
\end{aligned}
$$

where $\lambda$ is the dislocation array period. Within linear elasticity theory, $u_{x x}^{0}$ and $u_{y y}^{0}$ are given by 23 .

$$
\begin{aligned}
& u_{x x}^{0}(x)=\frac{b z}{2 \pi(1-\nu)} \frac{\left(3 x^{2}+z^{2}\right)}{\left(x^{2}+z^{2}\right)^{2}}, \\
& u_{y y}^{0}(x)=\frac{b z \nu}{\pi(1-\nu)} \frac{1}{x^{2}+z^{2}} .
\end{aligned}
$$

Here $z$ is the distance from the dislocation plane, $\nu$ is the Poisson's ratio and $b$ is the magnitude of the Burger's vector b. Similarly, the set of dislocation lines along the $x$ direction creates strain fields $\tilde{u}_{x x}$ and $\tilde{u}_{y y}$, which are related to $u_{x x}$ and $u_{y y}$ by the $\pi / 2$ rotation: $\tilde{u}_{x x}(y)=$ $u_{y y}(x \rightarrow y)$ and $\tilde{u}_{y y}(y)=u_{x x}(x \rightarrow y)$.

The total strain fields $u_{x x}(x)+\tilde{u}_{x x}(y)$ and $u_{y y}(x)+$ $\tilde{u}_{y y}(y)$ create gauge fields for the two-dimensional Dirac fermions at the interface. It follows from Eq. (1) that the gauge field $\mathbf{A}_{j}$ for the Dirac fermion at $\mathbf{K}_{j}$ contains both longitudinal and transverse components, $\mathbf{A}_{j}=\mathbf{A}_{j}^{L}+\mathbf{A}_{j}^{T}$. The longitudinal component $\mathbf{A}_{j}^{L}$ can be "gauged away" by a unitary transformation hence will not be considered below. The transverse component $\mathbf{A}_{j}^{T}$ is given by

$$
\begin{aligned}
& \mathbf{A}_{1}^{T}(y)=\left(\alpha_{1} \tilde{u}_{x x}(y)+\alpha_{2} \tilde{u}_{y y}(y), 0\right), \\
& \mathbf{A}_{2}^{T}(x)=\left(0, \alpha_{1} u_{y y}(x)+\alpha_{2} u_{x x}(x)\right),
\end{aligned}
$$

which yields an out-of-plane pseudo-magnetic field $B_{j}$ acting on the Dirac fermion at valley $\mathbf{K}_{j}: B_{1}(y)=$ $\nabla \times \mathbf{A}_{1}^{T}(y)$ and $B_{2}(x)=\nabla \times \mathbf{A}_{2}^{T}(x)$. Note that although the dislocation array is two-dimensional, the pseudomagnetic field for a given Dirac valley is periodically alternating in one direction only.

We now estimate the magnitude of the pseudomagnetic field created by misfit dislocation arrays at the interface. For a typical array period of $\lambda=15 \mathrm{~nm}$ at $z=$ $2 \mathrm{~nm}$, with the Poisson ratio for PbTe of $\nu=0.26[24]$ and the lattice constant $a=6.4 \AA$, the corresponding strain field $u=u_{x x}+u_{y y}$, plotted in Fig, 2 , has a maximum value of $6 \%$, which is comparable to the $3-10 \%$ lattice 

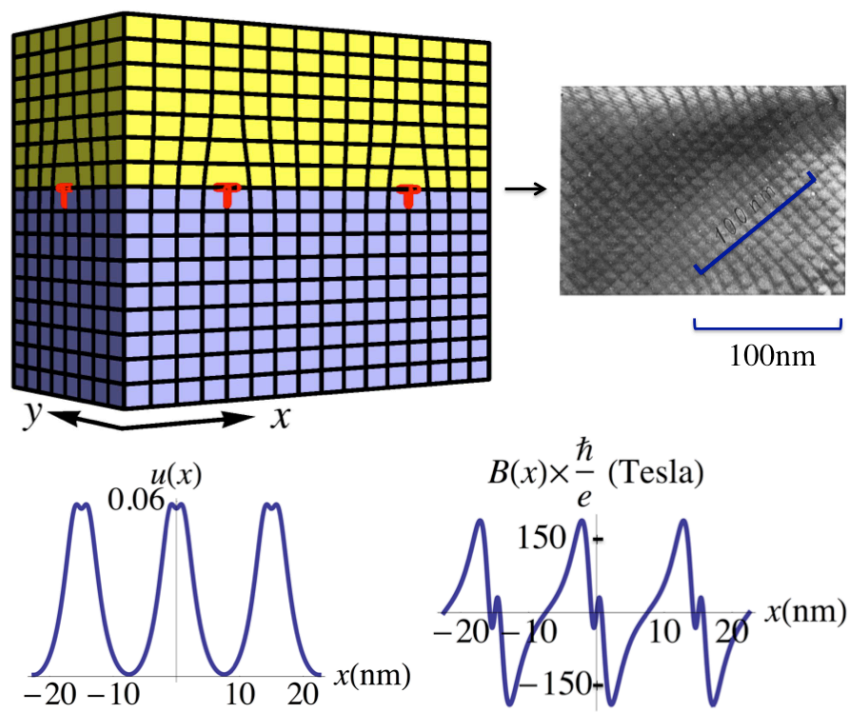

FIG. 2. The spontaneous formation of a misfit dislocation array gives rise to a periodically varying strain field and pseudo-magnetic field, at the interface. Top: a square array of misfit edge dislocations is spontaneously formed at the (001) interface of two IV-VI semiconductors (e.g., $\mathrm{PbTe} / \mathrm{PbSe}$ ), due to lattice mismatch. The array consists of dislocation lines along both $x$ and $y$ directions, as shown in the transmission electron microscopy image taken from Ref. 33. Bottom: the set of dislocation lines along the $y$ direction creates a periodically varying strain field $u(x) \equiv u_{x x}(x)+u_{y y}(x)$ as a function of $x$ given by Eq. (3). This is plotted here using the realistic parameters: $\lambda=15 \mathrm{~nm}$, $z=2 \mathrm{~nm}, \nu=0.26$ and $a=6.4 \AA$ (see main text), together with the pseudo-magnetic field $B(x)$ it generates.

mismatch in IV-VI semiconductor heterostructures 2022]. A recent ab-initio calculation[25] finds that the Dirac point positions of strained PbTe in the topological crystalline insulator phase shift linearly under compression, and yields $\alpha_{1}=2.2 \AA^{-1}$. Assuming $\alpha_{2} \approx \alpha_{1} \equiv \alpha$, we find a Dirac point displacement of $A_{0}=0.13 \AA^{-1}$ under the maximum $6 \%$ strain, which is comparable to the displacement under changes in alloy composition in $\mathrm{Pb}_{1-x} \mathrm{Sn}_{x}$ Te as recently observed by angle-resolved photoemission spectroscopy [15]. Using this value of $\alpha$, we plot the periodically alternating pseudo-magnetic field created by the dislocation array in Fig 2 . The maximum field strength is around 180T.

How does this pseudo-magnetic field change the electronic structure of Dirac fermion surface states at the interface? We first analyze this problem using a local field approximation. When the magnetic field is uniform, twodimensional massless Dirac fermions form a set of Landau levels. In the Landau gauge, the Landau orbitals are onedimensional strips localized at different $x$ positions and infinite in the $y$ direction. The width of the strip is set by the magnetic length $L_{B}=1 / \sqrt{|B|}$. When the magnetic field $B(x)$ is slowly varying over the distance $L_{B}$, the Landau level strip remains an approximate energy eigenstate. However, the Landau level energy becomes position dependent and is determined by the local magnetic field:

$$
E_{n}(x)=\operatorname{sgn}(n) \sqrt{2 n v_{x} v_{y}|B(x)|} .
$$

where $v_{x}\left(v_{y}\right)$ is the Dirac fermion velocity in the $x(y)$ direction. Since the $x$ position of a Landau level strip is proportional to its momentum in the $y$ direction i.e. $x \propto \operatorname{sgn}(B) k_{y}$, the Landau level energy $E_{n}(x)$ as a function of position $x$ is also the dispersion as a function of momentum $k_{y}$. Thus, when the magnetic field is spatially varying, Landau levels at different positions become nondegenerate and collectively form a dispersive band. It should be clear from this analysis that the extensive degeneracy at $E=0$ holds so long as the strain field varies slowly over $L_{B}$ even without being strictly periodic.

The $n=0$ Landau level of massless Dirac fermions deserves special attention. Its energy is pinned at $E_{0}=0$ independent of the magnetic field strength, as recently observed in topological crystalline insulators [5. This implies that in a slowly varying pseudo-magnetic field, the $n=0$ Landau orbitals remain extensively degenerate at zero energy, forming a flat band.

To demonstrate this, we numerically calculate the energy spectrum of massless Dirac fermions under the periodically alternating pseudo-magnetic field created by the dislocation array. The Hamiltonian is given by

$$
H=-i v_{x} \partial_{x} s_{y}-v_{y}\left(k_{y}-A_{y}(x)\right) s_{x},
$$

where $s_{x}$ and $s_{y}$ are Pauli matrices. Here we have chosen to study the Dirac valley $\mathbf{K}_{2}$, for which the strain-induced gauge field $A_{y}(x)$ is given in Eq. (4). For simplicity, we approximate the strain field shown in Fig 2 as a cosine function in our calculation, i.e., $A_{y}(x)=A_{0} \cos (2 \pi x / \lambda)$. The main findings presented below are independent of the specific choice of $A_{y}(x)$.

It is instructive to express $H$ in terms of the dimensionless quantities $\tilde{x} \equiv x / \lambda, \tilde{y} \equiv v_{x} y / v_{y} \lambda$ and $\tilde{H} \equiv \lambda H / v_{x}$ :

$$
\tilde{H}=-i \partial_{\tilde{x}} s_{y}-\left(\tilde{k}_{y}-\beta \cos (2 \pi \tilde{x})\right) s_{x} .
$$

The energy spectrum of $\tilde{H}$ is entirely determined by a single dimensionless parameter $\beta=\left(v_{y} / v_{x}\right) A_{0} \lambda \propto \sqrt{\lambda / L_{B}}$, which depends on the ratio of the dislocation array period and the magnetic length. The formation of local Landau levels and flat bands requires $\beta \gg 1$. To estimate the realistic value of $\beta$, we use the aforementioned parameter $A_{0}=0.13 \AA^{-1}$, and take Dirac velocities from an ab-initio calculation [12]: $v_{x}=0.84 \mathrm{eV} \AA, v_{y}=1.3 \mathrm{eV} \AA$. This yields $\beta \approx 20-40$ for $\lambda=10-20 \mathrm{~nm}$, fulfilling the condition for flat bands.

A representative band structure of $H$ for $\beta=30$ is plotted in Fig. 3a. As expected, we find two bands become very flat at zero energy for $\left|k_{y}\right|<k_{c}=0.05 \AA^{-1}$. These two degenerate flat bands correspond to two types 
a)

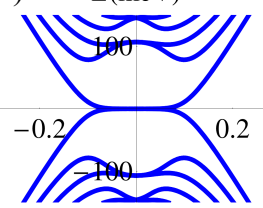

c)
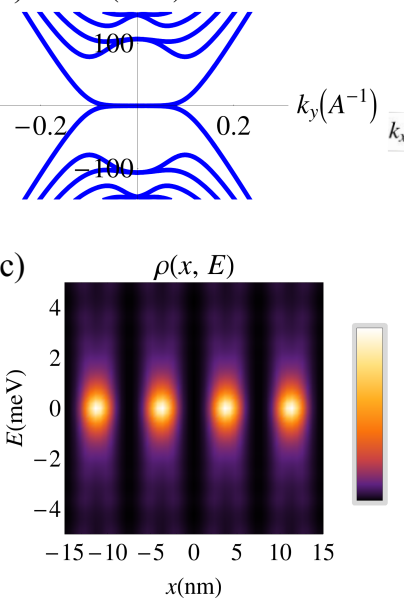

b)

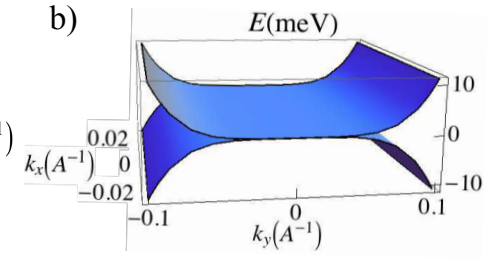

d)

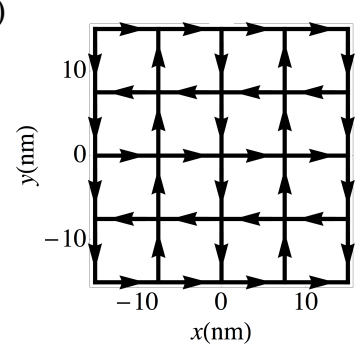

FIG. 3. The pseudo-magnetic field from strain creates flat bands and snake states. (a) Under a periodically alternating pseudo-magnetic field $B(x)$, the initially linear Dirac dispersion becomes flat within a finite range of $k_{y}$ in the vicinity of the Dirac point, while higher Landau levels are more dispersive. (b) The band is completely flat in $k_{x}$, which has a much reduced period given by the dislocation superlattice Brillouin zone. (c) Local density of states $\rho(x, E)$ as a function of position $x$ and energy $E$, showing zero-energy Landau levels from regions of both positive and negative pseudo-magnetic field, which are spatially separated by dispersive snake states. (d) One-dimensional snake states appear where the pseudo-magnetic field changes sign. This schematic cartoon shows snake states from valleys $\mathbf{K}_{1}$ and $\mathbf{K}_{2}$ moving along the $y$ and $x$ directions respectively, which form a two-dimensional network. Please refer to the main text for parameters used.

of $n=0$ Landau level strips that reside in different spatial regions under positive and negative pseudo-magnetic fields respectively. The $x$ position of Landau level strips in $B>0(B<0)$ regions increases (decreases) linearly with $k_{y}$. For $\left|k_{y}\right|>k_{c}$, these two types of Landau levels become hybridized in "transition regions" where $B$ goes through zero, which are centered either on a dislocation line at $x=N \lambda$ or at the midpoint $x=\left(N+\frac{1}{2}\right) \lambda$ between adjacent dislocations. As a consequence of the level splitting from hybridization, the two zero-energy flat bands at $\left|k_{y}\right|<k_{c}$ evolve into a pair of dispersive bands at $\left|k_{y}\right|>k_{c}$, which reside on the domain wall between $n=0$ Landau levels under positive and negative pseudo-magnetic fields (see Fig. 3p).

These dispersive states have a topological origin related to the unique half-integer Hall conductance of the $n=0$ Landau level of a single massless Dirac fermion: $\sigma_{x y}=\operatorname{sgn}(\mu B) \frac{1}{2} \frac{e^{2}}{h}$, where $\mu$ is the chemical potential. Because Hall conductances in $B>0$ and $B<0$ regions differ by $\Delta \sigma_{x y}=\operatorname{sgn}(\mu) e^{2} / h$, there exists one branch of chiral snake states at the domain wall 27, which is derived from the valley $\mathbf{K}_{2}$ and moves parallel to the $y$ axes.

The existence of these snake states, which is required by topology, leads to the electron-hole symmetric dispersion at large $k_{y}$ shown in Fig. 3a. The opposite velocities of snake states at $\mu>0$ and $\mu<0$ are required by the sign reversal of $\sigma_{x y}$.

The above topological flat bands and snake states derived from the Dirac valley at $\mathbf{K}_{2}$ have a time-reversed partner from the opposite valley at $-\mathbf{K}_{2}$ with opposite spin polarizations, as well as $\pi / 2$-rotated copies from the two other valleys at $\pm \mathbf{K}_{1}$. Taking all four valleys into account, we conclude that the dislocation array causes dramatic band reconstruction on the (001) surface by creating flat dispersions in the vicinity of the Dirac points and a two-dimensional network of helical snake states at higher energy. Due to the helical nature of topological surface states, counter-propagating snake states on the same domain wall cannot backscatter, and the twodimensional surface of topological crystalline insulators remains conducting even in the presence of disorder 35, unlike non-topological flat bands that are prone to Anderson localization.

Recently flat-band systems have attracted tremendous interest due to enhanced interaction effects associated with the high density of states and the resulting electronic instabilities. Most studies have focused on finding a completely flat band with nontrivial topology, which appears to rely on careful fine tuning of material parameters and has not been experimentally realized so far. In comparison, our work reveals a new and realistic route to achieve a partially flat band, as shown in Fig.3. For a wide range of electron or hole densities (up to about $5 \times 10^{12} \mathrm{~cm}^{-2}$ in this example), the Fermi energy lies within the partially filled flat band. It is thus natural to ask whether interesting interaction-driven phenomena are expected to arise.

In this regard, we note that a wide class of IVVI semiconductor (001) multilayers and bilayers is superconducting. Superconductivity was first discovered in $\mathrm{PbTe} / \mathrm{SnTe}, \mathrm{PbSe} / \mathrm{PbS}, \mathrm{PbTe} / \mathrm{PbSe}, \mathrm{PbS} / \mathrm{YbS}$ and $\mathrm{PbTe} / \mathrm{YbS}$ superlattices long ago 28 30, and recently found in two-layer sandwiches of $\mathrm{PbTe} / \mathrm{PbS}, \mathrm{PbTe} / \mathrm{PbSe}$, and $\mathrm{PbTe} / \mathrm{YbS}$ with layers $40-300 \mathrm{~nm}$ thick 31, 32. The transition temperatures $T_{c}$ are in the range of $2.5-6.4 \mathrm{~K}$, which is unusually high for semiconductors especially given that the individual constituent materials are nonsuperconducting (the only exception is SnTe with the very low $T_{c}$ of $0.22 \mathrm{~K}$ ). Further, the strong anisotropy of the upper critical field reveals that the observed superconductivity is two-dimensional [31, 32]. Based on these facts, it was concluded that the locus of superconductivity is at the interface.

Remarkably, it was found that the appearance of superconductivity is dependent on the formation of a misfit dislocation array near the interface 33, 34. Samples with island-type growth, and therefore dislocations that do not cover the whole interface only show partial su- 
perconducting transitions. For superconducting samples, the transition temperature $T_{c}$ was found to increase from $3 \mathrm{~K}$ to $6 \mathrm{~K}$ as the period of the misfit dislocation array $\lambda$ decreases from $23 \mathrm{~nm}$ to $10 \mathrm{~nm} 33$.

Previous works 30, 33 have proposed that superconductivity emerges from metallic interface states created by band inversion on one side of the interface where the constituent material is pseudomorphic and in compression. Such band inversion due to compression is only possible for narrow-gap semiconductors, which explains the absence of superconductivity down to $1.5 \mathrm{~K}$ in IV-VI multilayers consisting of only wide-gap semiconductors (YbS/EuS, YbS/YbSe). However, this proposal does not take into account the indispensable role of dislocations in superconductivity, nor explain the origin of the unusually high transition temperature.

Our work sheds new light on the interface superconductivity in IV-VI multilayers. The band inversion induced by compression leads to the topological crystalline insulator phase [1, and hence gives rise to topological surface states at the interface whose electronic properties were correctly identified only recently[1,4, 12]. At the same time, the dislocation array produces a periodically varying strain, which acts on these states to create topological flat bands. In the presence of attractive interaction due to electron-phonon coupling, the high density of states associated with these flat bands dramatically increases the superconducting transition temperature.

Our proposal of interface superconductivity from dislocation-induced flat bands provides a remarkable explanation of the unusual dependence of $T_{c}$ on the dislocation array period 33 . As shown recently, the superconducting transition temperature $T_{c}$ in a flat-band system is linearly proportional to the area of the flat band in momentum space 36, 37, which is parametrically enhanced compared to BCS theory. In our proposal, when the distance between dislocation lines is large, the pseudomagnetic field created by the strain field (3) is largely concentrated around individual dislocation lines, thus the zero-energy Landau orbitals from different dislocations are spatially separated. In this regime, the area of the flat bands in momentum space is proportional to the reciprocal superlattice vector $2 \pi / \lambda$, and thus $T_{c}$ increases with decreasing array period. When $\lambda$ becomes too small, however, Landau orbitals start to overlap and band flatness gets destroyed, hence $T_{c}$ stops increasing. In effect, the dependence of $T_{c}$ on the flat band degeneracy results in its non-monotonic dependence on the array period, in agreement with the experimental observation 33 .

We further predict two testable signatures of flat bands in topological crystalline insulators and their prominent role in interface superconductivity in IV-VI semiconductor multilayers. First, the flat bands and coexisting network of helical snake states generate a distinctive local density of states spectrum as a function of position and energy shown in Fig.3c, which can be detected in tunneling (magneto-)conductance measurements [38, de Haas-van Alphen or Shubnikov-de Haas oscillations. Second, the enhancement of superconductivity by flat bands ceases to work when the flat bands become empty or filled. This leads to a drop in $T_{c}$ as the carrier density at the interface increases above a threshold, whose value depends on the strain field strength and dislocation array period and is estimated to be on the order of $10^{12} \mathrm{~cm}^{-2}$.

While the superconducting transition temperatures in IV-VI heterostructures are not high on an absolute scale, the mechanism of flat band formation due to interface microstructures or intentional strain engineering may offer a viable route to high-temperature interface superconductivity - a subject of tremendous current interest [39, 40. Further, our work opens up new directions for achieving other interesting phases in a realistic setting. In particular, when interactions are repulsive 4143, novel quantum Hall states or fractional topological insulators may arise in the helical flat band we have found, at zero magnetic field. While electron repulsion is weak in IV-VI semiconductors due to their large dielectric constants, new topological crystalline insulator materials have recently been predicted/proposed in correlated electron systems such as heavy fermion compounds 44, 45, transition metal oxides 46, graphene multilayers 47 and anti-perovskites [48]. These rapid and continuing developments hold promise for the physical realization of new states of matter in partially flat bands.

\section{METHODS}

Here we provide a derivation of the strain-induced gauge field (1), or equivalently Dirac point displacement on the (001) surface Brillouin zone of topological crystalline insulators. The derivation is based on symmetry analysis. A generic in-plane strain $u_{i j}$ can be decomposed into three independent components: compression/dilation $u_{x x}+u_{y y}$, uniaxial stretch $u_{x x}-u_{y y}$, and shear $u_{x y}+u_{y x}$, which transform differently under crystal symmetries.

Compression/dilation preserves the full symmetry of the (001) surface. In particular, the presence of two mirror planes (110) and (110) guarantees the two pairs of Dirac points $\pm \mathbf{K}_{1}$ and $\pm \mathbf{K}_{2}$ lie along the mirror-invariant lines $\Gamma X_{1}$ and $\Gamma X_{2}$ respectively, in the surface Brillouin zone 11. Importantly, the Dirac point positions on the $\Gamma X$ lines are not constrained by symmetry [12]; they vary continuously under strain[49]. As shown in recent abinitio calculations 25, 26, a compressive (tensile) strain moves $\pm \mathbf{K}_{1}$ and $\pm \mathbf{K}_{2}$ towards (away from) the Brillouin zone center $\Gamma$ (see Fig.1b).

Uniaxial stretch in the [11̄0] direction preserves both (110) and (110) mirror planes but breaks the four-fold symmetry: $u_{x x}-u_{y y}$ is odd under the $\pi / 2$ rotation $x \rightarrow$ $y, y \rightarrow-x$. As a consequence, the Dirac points $\pm \mathbf{K}_{1}$ 
and $\pm \mathbf{K}_{2}$ move along the $\Gamma X$ lines by an equal distance but in opposite directions: $\pm \mathbf{K}_{1}$ move inward and $\pm \mathbf{K}_{2}$ move outward (see Fig.1c).

Shear strain breaks both (110) and (110) mirror symmetries, as well as the four-fold rotation symmetry. Therefore, Dirac points move perpendicular to the $\Gamma X$ lines, and the displacement vector $\mathbf{K}_{1}^{\prime}-\mathbf{K}_{1}$ is opposite to $\mathbf{K}_{2}^{\prime}-\mathbf{K}_{2}$ after the $\pi / 2$ rotation (see Fig.1d).

It follows from the above analysis that a generic straininduced gauge field $\mathbf{A}_{j}$ acting on the Dirac fermion at valley $\mathbf{K}_{j}$ consists of contributions from compression/dilation, stretch and shear, which involve three independent coupling constants. Adding up the corresponding Dirac point displacements leads to the expression of $\mathbf{A}_{j}$ in (1). Note that the form of $\mathbf{A}_{j}$ in topologically crystalline insulators is different from its counter-part in graphene, which has been extensively studied in recent years 16 19]. For example, unlike here, a dilation in graphene does not generate a gauge field due to the pinning of Dirac points at Brillouin zone corners. This difference arises from the important distinction in crystal symmetry and electronic topology.

\section{ADDITIONAL INFORMATION}

Correspondence and requests for materials should be addressed to LF.

\section{ACKNOWLEDGEMENTS}

We thank Maksym Serbyn and Andrea Allais for helpful discussions, as well as Yoichi Ando and Robert Cava for valuable comments on the manuscript. This work is supported by DOE Office of Basic Energy Sciences, Division of Materials Sciences and Engineering under award de-sc0010526 (LF). ET acknowledges support from NSF Grants DMR-1005541, NSFC 11074140, and NSFC 11274192.

\section{AUTHORS CONTRIBUTION}

Both authors contributed to theoretical and numerical parts of the research, and the writing of the manuscript.

[1] T. H. Hsieh, H. Lin, J. Liu, W. Duan, A. Bansil and L. $\mathrm{Fu}$, Topological crystalline insulators in the SnTe material class, Nat. Commun. 3, 982 (2012).

[2] Y. Tanaka, Zhi Ren, T. Sato, K. Nakayama, S. Souma, T. Takahashi, K. Segawa and Y. Ando, Experimental realization of a topological crystalline insulator in SnTe, Nat. Phys. 8, 800-803 (2012).
[3] P. Dziawa, B. J. Kowalski, K. Dybko, R. Buczko, A. Szczerbakow, M. Szot, E. Lusakowska, T. Balasubramanian, B. M. Wojek, M. H. Berntsen, O. Tjernberg and T. Story, Topological crystalline insulator states in $\mathrm{Pb}_{1 x} \mathrm{Sn}_{x}$ Se, Nat. Mat. 11, 1023-1027 (2012).

[4] S. Y. Xu, C. Liu, N. Alidoust, M. Neupane, D. Qian, I. Belopolski, J. D. Denlinger, Y. J. Wang, H. Lin, L. A. Wray, G. Landolt, B. Slomski, J.H. Dil, A. Marcinkova, E. Morosan, Q. Gibson, R. Sankar, F.C. Chou, R.J. Cava, A. Bansil and M.Z. Hasan, Observation of a topological crystalline insulator phase and topological phase transition in $\mathrm{Pb}_{1 x} \mathrm{Sn}_{x}$ Te, Nat. Commun. 3, 1192 (2012).

[5] Y. Okada, M. Serbyn, H. Lin, D. Walkup, W. Zhou, C. Dhital, M. Neupane, S. Xu, Y. Wang, R. Shankar, F. Chou, A. Bansil, M. Hasan, S. Wilson, L. Fu and V. Madhavan, Observation of Dirac node formation and mass acquisition in a topological crystalline insulator, Science, 341, 1496-1499 (2013).

[6] J. Liu, T. H. Hsieh, P. Wei, W. Duan, J. Moodera and L. $\mathrm{Fu}$, Spin-filtered Edge States with an Electrically Tunable Gap in a Two-Dimensional Topological Crystalline Insulator, Nat. Mat. 13, 178183 (2014).

[7] C. Fang, M. Gilbert and B. A. Bernevig, Large Chern Number Quantum Anomalous Hall Effect In Thin-film Topological Crystalline Insulators, Phys. Rev. Lett. 112, 046801 (2014).

[8] F. Zhang, X. Li, J. Feng, C. L. Kane and E. J. Mele, Zeeman Field-Tuned Transitions for Surface Chern Insulators, arXiv:1309.7682

[9] M. Z. Hasan and C. L. Kane, Topological Insulators, Rev. Mod. Phys. 82, 3045 (2010).

[10] X. L. Qi, and S. C. Zhang, Topological insulators and superconductors, Rev. Mod. Phys. 83, 1057 (2011).

[11] J. E. Moore, The birth of topological insulators, Nature 464, 194-198 (2010).

[12] J. Liu, W. Duan and L. Fu, Surface States of Topological Crystalline Insulators in IV-VI Semiconductors, Phys. Rev. B 88, 241303(R) (2013).

[13] L. Fu, Topological crystalline insulators, Phys. Rev. Lett. 106, 106802 (2011).

[14] R. S. K. Mong, A. M. Essin and J. E. Moore, Antiferromagnetic topological insulators, Phys. Rev. B 81, 245209 (2010).

[15] Y. Tanaka, T. Sato, K. Nakayama, S. Souma, T. Takahashi, Z. Ren, M. Novak, K. Segawa, Y. Ando, Tunability of the $k$-space location of the Dirac cones in the topological crystalline insulator $\mathrm{Pb}_{1 x} \mathrm{Sn}_{x}$ Te, Phys. Rev. B 87, 155105 (2013).

[16] N. Levy, S. A. Burke, K. L. Meaker, M. Panlasigui, A. Zettl, F. Guinea, A. H. Castro Neto and M. F. Crommie, Strain-Induced Pseudo-Magnetic Fields Greater Than 300 Tesla in Graphene Nanobubbles, Science, 329, 544547 (2010).

[17] J. L. Mañes, Symmetry-based approach to electronphonon interactions in graphene, Phys. Rev. B 76, 045430 (2007).

[18] F. Guinea, M. I. Katsnelson and A. K. Geim, Energy gaps and a zero-field quantum Hall effect in graphene by strain engineering, Nat. Phys. 6, 30-33 (2009).

[19] V. M. Pereira and A. H. Castro Neto, Strain Engineering of Graphenes Electronic Structure, Phys. Rev. Lett. 103, 046801 (2009).

[20] A. Yu. Sipatov, Superlattice nanostructures based on chalcogenide materials, Functional Materials, 16, 374- 
382 (2009).

[21] L. S. Palatnik and A. I. Fedorenko, Formation of dislocation superlattices in epitaxial systems, J. Cryst. Growth 52, 917-924 (1981).

[22] G. Springholz and K. Wiesauer, Nanoscale Dislocation Patterning in $\mathrm{PbTe} / \mathrm{PbSe}(001)$ Lattice-Mismatched Heteroepitaxy, Phys. Rev. Lett. 88, 015507 (2001).

[23] P. Chaikin and C. L. Lubensky, Principles of Condensed Matter Physics, Cambridge University Press (2000).

[24] M. J. Weber, Handbook of Optical Materials, CRC Press (2002).

[25] P. Barone, D. D. Sante and S. Picozzi, Strain engineering of topological properties in lead-salt semiconductors, Phys. Status Solidi RRL 7, 1102-1106 (2013).

[26] X. Qian, L. Fu and J. Li, Topological Crystalline Insulator Nanomembrane with Strain-Tunable Band Gap, arXiv:1403.3952

[27] A network of chiral snake states in a real magnetic field was studied in D. B. Chklovskii and P. A. Lee, Transport properties between quantum Hall plateaus, Phys. Rev. B 48, 18060 (1993).

[28] K. Murase, S. Ishida, S. Takaoka, and T. Okumura, Superconducting behavior in PbTe-SnTe superlattices, Surf. Sci. 170, 486-490 (1986).

[29] O. A. Mironov, A. B. Savitskii, A. Yu. Sipatov, A. I. Fedorenko, A. N. Chirkin, S. V. Chistyakov, and L. P. Shpakovskaya, Superconductivity of semiconductor superlattices based on lead chalcogenides, JETP Lett. 48, 106-109 (1988).

[30] D. Agassi and T. K. Chu, Strain Induced Superconductivity in Lead Salt Superlattices, Phys. Status Sol. (b) 160, 601-611 (1990).

[31] N. Ya. Fogel, E. I. Buchstab, Yu. V. Bomze, O. I. Yuzephovich, M. Yu. Mikhailov, A. Yu. Sipatov, E. A. Pashitskii, R. I. Shekhter, and M. Jonson, Direct evidence for interfacial superconductivity in two-layer semiconducting heterostructures, Phys. Rev. B 73, 161306(R) (2006).

[32] O. I. Yuzephovich, M. Yu. Mikhailov, S. V. Bengus, A. Yu. Aladyshkin, E. E. Pestov, Yu. N. Nozdrin, A. Yu. Sipatov, E. I. Buchstab, and N. Ya. Fogel, Interfacial superconductivity in bilayer and multilayer IVVI semiconductor heterostructures, Low Temp. Phys. 34, 985 (2008).

[33] N. Ya. Fogel, E. I. Buchstab, Yu. V. Bomze, O. I. Yuzephovich, A. Yu. Sipatov, E. A. Pashitskii, A. Danilov, V. Langer, R. I. Shekhter, and M. Jonson, Interfacial superconductivity in semiconducting monochalcogenide superlattices, Phys. Rev. B 66, 174513 (2002).

[34] N. Ya. Fogel, A. S. Pokhila, Yu. V. Bomze, A. Yu. Sipatov, A. I. Fedorenko, and R. I. Shekhter, Novel Super- conducting Semiconducting Superlattices: DislocationInduced Superconductivity?, Phys. Rev. Lett. 86, 512 (2001).

[35] L. Fu and C. L. Kane, Topology, Delocalization via Average Symmetry and the Symplectic Anderson Transition, Phys. Rev. Lett. 109, 246605 (2012).

[36] N. B. Kopnin, T. T. Heikkila and G. E. Volovik, Hightemperature surface superconductivity in topological flat-band systems, Phys. Rev. B 83, 220503(R) (2011).

[37] V. Khodel and V. Shaginyan, Topological origin of zeroenergy edge states in particle-hole symmetric systems, JETP Lett. 51, 553, 1990.

[38] R. Yoshimi, A. Tsukazaki, K. Kikutake, J. G. Checkelsky, K. S. Takahashi, M. Kawasaki and Y. Tokura, Dirac electron states formed at the heterointerface between a topological insulator and a conventional semiconductor, Nat. Mat. 13, 253257 (2014).

[39] J. Pereiro, A. Petrovic, C. Panagopoulos and I. Bozovic, Interface superconductivity: History, development and prospects, Physics Express, 1, 208-241 (2011).

[40] W. Zhang et al, Direct observation of high temperature superconductivity in one-unit-cell FeSe films, Chin. Phys. Lett. 31, 017401 (2014).

[41] P. Ghaemi, J. Cayssol, D. N. Sheng, A. Vishwanath, Fractional topological phases and broken time reversal symmetry in strained graphene, Phys. Rev. Lett. 108, 266801 (2012).

[42] M. Levin and A. Stern, Fractional Topological Insulators, Phys. Rev. Lett. 103, 196803 (2009).

[43] B. Roy, F. F. Assaad and I. F. Herbut, Zero Modes and Global Antiferromagnetism in Strained Graphene, Phys. Rev. X 4, 021042 (2014).

[44] H. Weng, J. Zhao, Z. Wang, Z. Fang and Xi Dai, Topological Crystalline Kondo Insulator in Mixed Valence Ytterbium Borides, Phys. Rev. Lett. 112, 016403 (2014).

[45] M. Ye, J. W. Allen and K. Sun, Topological crystalline Kondo insulators and universal topological surface states of $\mathrm{SmB}_{6}$, arXiv:1307.7191

[46] M. Kargarian and G. A. Fiete, Topological Crystalline Insulators in Transition Metal Oxides, Phys. Rev. Lett. 110, 156403 (2013).

[47] M. Kindermann, Topological crystalline insulator phase in graphene multilayers, arXiv:1309.1667

[48] T. H. Hsieh, J. Liu and L. Fu, Topological Crystalline Insulators and Dirac Octets in Anti-perovskites, arXiv:1407.4809

[49] M. Serbyn and L. Fu, Symmetry breaking and Landau levels in a topological crystalline insulator, Phys. Rev. B 90, 035402 (2014). 